\title{
TWO WORKS ON ITERATION AND RELATED QUESTIONS
}

\section{J. HADAMARD}

I want to give a brief account of results which have been communicated to me and which have been obtained by two young geometers, Eri Jabotinsky and Michel Lüntz. Mr. Lüntz is unhappily in a concentration camp in France. I must add that this is one of the reasons for the fact, for which I must apologize, that I am writing this expose only now, although I have had both works in my possession for several months. The impossibility of communicating with $\mathrm{Mr}$. Lüntz and therefore of asking him for any explanation made the examination of his paper especially difficult.

As is well known, the problem of iteration, a function $f(x)$ being given, consists in finding a one-parameter family of functions $f_{n}(x)$ $=f(n, x)$ such that, for $n=1$, we have

$$
f(1, x)=f(x)
$$

and, moreover, for any $m, n$,

$$
f_{m}\left[f_{n}(x)\right]=f_{m+n}(x)
$$

also

$$
f(0, x)=x
$$

which follows from (2) as is seen by taking $m=0$.

The question is connected with Abel's functional equation

$$
\phi[f(x)]=1+\phi(x)
$$

because if $\phi$ is a solution of that equation a solution of (1), (2) will be given by

$$
\phi\left[f_{n}(x)\right]=n+\phi(x) .
$$

Instead of $(A)$, one can introduce Schroeder's equation

$$
\psi[f(x)]=k \psi(x)
$$

( $k$ a constant) in which $\psi$ is connected with the unknown $\phi$ of (A) by $\psi=k^{\phi}$, and with the help of which the solution would be expressed by

$$
\psi\left[\left(f_{n}(x)\right]=k^{n} \psi(x) .\right.
$$

The fact that every solution of (A) gives a solution of the iteration

Presented to the Society, April 24, 1943, under the title On fractional iteration and connected questions; received by the editors July 1, 1943. 
problem is sufficient to show that the latter is indeterminate unless proper conditions are added: for, if $\phi(x)$ is a solution of (A), so is also $\phi(x)+\Pi[\phi(x)]$ where $\Pi$ is any function admitting of the period 1 .

The iteration problem has been considered by several authors. The most recent work, as far as I know, is due to Paul Lévy (C. R. Acad. Sci. Paris vol. 184 p. 500 and Annali di Matematica (4) vol. 5 (1928) pp. 270-296, esp. p. 287 ff.). Additional conditions being necessary (as a matter of fact, conditions of regularity), Paul Lévy imposes such conditions either (1) at infinity-a difficult subject, which we shall leave aside-or (2) in the neighborhood of a determinate value, which the successive iteratives of $f$ are assumed to approach. In the latter case, the one which will interest us, he uses the classic studies of Koenigs (Ann. Ecole Norm. 1884).

One feature of the problem is not considered by the above mentioned authors, namely that, on account of (2), the set of the various transformations $x \rightarrow f(n, x)$ is a one-parameter group, which can be defined by its infinitesimal transformation $\delta x=\xi(x) \delta t$. The question has been faced from this point of view in a note of Bouton ${ }^{1}$ for the case in which the transformations are assumed to be tangent to the identical one. Moreover, that group aspect and the infinitesimal transformation have also been introduced in the fundamental memoir of G. D. Birkhoff, ${ }^{2}$ though without playing an essential role.

Jabotinsky begins by considering not one problem of iteration, but the general class of them, that is, the equations (2) and (3) without immediate consideration of any special function $f(x)$ nor, therefore, of equation (1). Now all these problems satisfy a common partial differential equation. For, writing (2) in the form

$$
f[(m-n), f(n, x)]=f(m, x)
$$

and expressing the fact that the left-hand member does not depend on $n$, Jabotinsky easily obtains the partial differential equation

$$
\left(\partial^{2} f / \partial n^{2}\right)(\partial f / \partial x)-\left(\partial^{2} f / \partial n \partial x\right)(\partial f / \partial n)=0 .
$$

Conversely, this equation admits of the two intermediate integrals

$$
\begin{gathered}
(\partial f / \partial n):(\partial f / \partial x)=\xi(x), \\
\partial f / \partial n=\eta(f)
\end{gathered}
$$

${ }^{1}$ Bull. Amer. Math. Soc. vol. 23 (1917) p. 75: A scanty note, the development of which has not been published as far as we know.

2 Acta Math. vol. 43 (1922) p. 1. As is well known, that memoir deals with transformations in two variables, the existence of an integral invariant being assumed. See also the paper of Daniel C. Lewis, Duke Math. J. vol. 5 (1939) p. 794 in connection with G. D. Birkhoff's ideas. 
$(\xi, \eta$, arbitrary functions) so that its general solution is

$$
f(n, x)=\chi[n+\phi(x)] \quad\left(\phi(x)=\int d x / \xi(x), \chi \text { arbitrary }\right) .
$$

If we add condition (3), we see that $\chi$ must be the inverse function of $\phi$, so that the above formula brings us back to (A). Thus we have a simple proof of the fact that not only every solution of (A) gives us a solution of (2), (3), but that, conversely, ${ }^{3}$ every solution of (2) corresponds to a solution of $(A)$.

The partial differential equation (E) is common to every problem of the kind which interests us, whatever the given function $f(x)$ may be.

In order to integrate that equation, (3) gives the condition $f(0, x)=x$; and, if we now introduce (1), we see that we are also given the values of $f(n, x)$ for $n=1$. It is a boundary problem of an unusual form; and, indeed, we know that our problem is not determinate if we do not add complementary conditions of regularity.

As was already done in the aforesaid works of Koenigs and Paul Lévy, Jabotinsky investigates what will happen in the neighborhood of a "double point," that is of a value of $x$-say $x=0$ - such that

$$
f(x)=x,
$$

and he limits himself to iterates $f(n, x)$ of $f(x)$ such that every $f(n, 0)$ is also equal to 0 , a hypothesis which, as we shall see, hardly diminishes generality. Moreover, he assumes $f$ to be expansible in powers of $x$ and tries to find a corresponding expansion for $f_{n}$, say

$$
f_{n}(x)=A_{1}(n) x+A_{2}(n) x^{2}+\cdots .
$$

This is what Paul Lévy has carried out in his note in the C. R. Acad. Sci. Paris for the case of $f(x)=e^{x}-1$ (of which Jabotinsky was not informed). For that purpose, Jabotinsky substitutes (9) into (E) or, preferably, into the intermediate integral (5). If $\xi(x)=\alpha_{1} x+\alpha_{2} x^{2}+\cdots$ were known, this would give, for the $A_{i}(n)$, the successive linear differential equations of the first order

$$
\begin{aligned}
& A_{1}^{\prime}(n)=\alpha_{1} A_{1}(n), \\
& A_{2}^{\prime}(n)=2 \alpha_{1} A_{2}(n)+\alpha_{2} A_{1}(n), \\
& A_{3}^{\prime}(n)=3 \alpha_{1} A_{3}(n)+2 \alpha_{2} A_{2}(n)+\alpha_{3} A_{1}(n),
\end{aligned}
$$

${ }^{3}$ Around a double point (which case we consider below), it is necessary to replace (A) by Schroeder's equation ( $\left.A^{\prime}\right)$, as does Koenig in the above cited memoir. 
to be integrated with the initial conditions $A_{1}(0)=1, A_{i}(0)=0$ for $i \geqq 2$-an elementary integration.

Now, on account of (3), $\xi(x)$ is nothing else than the derivative of $f(n, x)$ with respect to $n$ for $n=0$, so that if $\xi$ were known, this would come back to solving Cauchy's problem for (E). From the point of view of group theory, $\xi(x)$ corresponds to the infinitesimal transformation of the group which interests us. ${ }^{4}$

As a matter of fact, in our case the $\alpha$ 's are auxiliary unknowns; they are to be determined by the knowledge of the values of the $A$ 's for $n=1$, which proceeds without any difficulty, for each $i$, after the integration of the successive equations (10). If $\alpha_{1} \neq 0$, that is if $a_{1}=A_{1}(1) \neq 1$, the expressions thus obtained obviously contain $n$ through polynomials in powers of $e^{\alpha_{1} n}=a_{1}{ }^{n}$, so that they are quite similar to those which G. D. Birkhoff has constructed in the case which he has investigated.

The case $a_{1}=1$ is comparable to Birkhoff's case $\mathrm{II}^{\prime \prime}$, the successive unknowns $A$ in (10) being polynomials in $n$.

But, as in Birkhoff's case, there remains the question whether the $A_{i}^{\prime}$ thus calculated would give a convergent series for (9); and, precisely, Birkhoff has shown, by a remarkable example (see §31, p. 55 of his memoir), that the contrary case may occur in the problem which he has treated. Whether the same fact is possible for the transformations in one variable remains to be decided.

Lüntz's starting point is slightly different: he investigates "mutually reversible" functions, two functions $f$ and $g$ being said to be reversible to each other ${ }^{5}$ if

$$
f[g(x)]=g[f(x)] .
$$

He points out that this is a kind of generalization of fractional iteration; and, indeed, we see at once by (2) that any two of the $f_{m}$ are reversible to each other. Conversely, will the investigation of func-

- If the transformation $x \rightarrow f(x)$ were known to belong to a certain group of Lie, $G_{r}$, in a finite number $r$ of parameters, our question would be considered as being treated by Elie Cartan in his memoir on Geométrie des groupes de transformations (Annali di Matematica (9) vol. 6 (1927) p. 1). In Cartan's terminology, a one-parameter group in $G_{r}$ is the analogue of a geodesic in the $G_{r}$-space, which would be defined by its origin (the identical transformation) and its initial direction (defined by the corresponding infinitesimal transformation); our problem would correspond to drawing a geodesic through two given points. The solution is elementary in Cartan's case, but of a quite different difficulty when no $G_{r}$ containing the given transformation is known.

- Lüntz is right in not using the word "permutable," which is used by Volterra in the Functional calculus with a different meaning. On the contrary, the transformations $x \rightarrow f(x)$ and $x \rightarrow g(x)$ are said to be permutable. 
tions $g$ reversible to a given function $f$ always lead to the iterates of $f$ ?

We shall presently see that, under the above postulated regularity conditions, such will be the case. But it must be noticed that this new problem is, from a certain point of view, simpler than the former since, instead of a family of functions, we investigate a single function $g$.

As was done above, Lüntz also starts from a double point $x_{0}$ of $f$, say $x_{0}=0$. Now, taking $x=x_{0}=0$ in the fundamental relation (11), we see that $g\left(x_{0}\right)$ must be a double point of $f$ also, so that Lüntz is led to admit that $g\left(x_{0}\right)=g(0)$ is nothing else than $x_{0}$ itself.

Lüntz also remarks that if $g_{1}$ and $g_{2}$ are both reversible to $f$, so is also $g_{1}\left[g_{2}(x)\right]$, so that (a well known fact in the transformation theory) the $g$ 's are the elements of a group. He, moreover, supposes that such functions $g_{1}$ and $g_{2}$ are also reversible to each other, which we shall see to be the case. ${ }^{6}$

Again, taking $x_{0}=0$ and representing $f$ and $g$ by expansions in powers of $x$,

(11) gives the relations

$$
\begin{aligned}
& a_{1} b_{1}=b_{1} a_{1}, \quad a_{1} b_{2}+a_{2} b_{1}^{2}=b_{1} a_{2}+b_{2} a_{1}^{2} \\
& a_{1} b_{3}+2 a_{2} b_{1} b_{2}+a_{3} b_{1}^{3}=b_{1} a_{3}+2 b_{2} a_{1} a_{2}+b_{1} a_{1}^{3}, \\
& \begin{array}{r}
a_{1} b_{4}+a_{2}\left(2 b_{1} b_{3}+b_{2}^{2}\right)+3 a_{3} b_{1}^{2} b_{2}+a_{4} b_{1}^{4} \\
\quad=b_{1} a_{4}+b_{2}\left(2 a_{1} a_{3}+a_{2}^{2}\right)+3 b_{3} a_{1}^{2} a_{2}+b_{4} a_{1}^{4}
\end{array}
\end{aligned}
$$

The first one being an identity, we see that if $a_{1}$ is neither equal to 0 (a case which the author excludes) nor to 1 , the coefficient $b_{1}$ is arbitrary and may be considered as an arbitrary parameter $\lambda$, by means of which we have the general form for $g$,

$$
\begin{aligned}
g(x) & =g_{\lambda}(x) \\
& =\lambda x+\left(\lambda-\lambda^{2}\right)\left\{\beta_{2} x^{2}+\left[\beta_{3}(1+\lambda)+\beta_{2}^{2}(1-\lambda)\right] x^{3}+\cdots\right\} .
\end{aligned}
$$

The one parameter family represented by the above expression for $g$ can not be distinct from the group (9), as every iterate of $f$ necessarily satisfies (11)

6 Lüntz notices the consequence that if $f(x)$ is odd-that is permutable with $x \rightarrow(-x)$ - so is also $g(x)$. 
Moreover, the above expression of the unknown is not written in terms of the $a$ 's but, as previously, implies an auxiliary set of parameters $\beta$, the meaning of which would not be obvious by itself, but appears by comparison with Jabotinsky's result. Indeed, giving the terms of the first degree in $\lambda-1$, they are, but for numerical factors, nothing else than the $\alpha$ in (10), so that the above formula leads us back to Jabotinsky's calculation.

Again, these parameters are to be found in terms of the $a$ 's, and the convergence question is left aside.

If we assume $a_{1}$ to be equal to 1 and if $a_{2} \neq 0$, the coefficient $b_{1}$ will no longer be arbitrary: it must be equal either to 0 or to 1 , so that the expansion of $g$ will be $\left(b_{1}=0\right.$ being again excluded) of the form $x+\cdots$, and will again include an arbitrary parameter $\lambda$, namely the coefficient $b_{2}$ of $x^{2}$.

The third, fourth, ... equations (14) allow us to calculate the successive $b$ 's. Again Lüntz is led to introduce a set of parameters which coincide with the coefficients in the infinitesimal transformation of the group and, therefore, to write the same formula as would be found by Jabotinsky's method.

Consequently, we see that for $a_{1} \neq 0,1$, as for $a_{2} \neq 0$, the family of functions reversible to $f$ and satisfying our regularity conditions is not distinct from the family of iterates of $f$.

These are the results obtained by Jabotinsky and Lüntz; they coincide with each other, at least if we do not have $a_{1}=0$ or $a_{1}=1$, $a_{2}=0$, though they are deduced from rather different points of view.

This coincidence suggests several remarks, some of which I shall point out now.

I. In what precedes, we have started from the hypothesis that a double point $x=x_{0}$ of $f(x)$ is common to all iterates $f(n, x)$ or to all the functions $g(x)$ reversible to $f$. As we have seen, Lüntz makes the important remark that, on account of the definition (11) of reversible functions, the transform $g\left(x_{0}\right)$ is also a double point of $f$. He leaves, however, one question open, namely whether this transform is $x_{0}$ itself or another solution of (8). But, as a matter of fact, this question can be answered easily if the function $g$ is assumed to belong to a continuous family of functions all of which are reversible to $f$, one of them being $g(x)=x$; for, substituting $x_{0}$ in each of these functions, we obtain results which, varying continuously, can not be different from $x_{0}$ itself, at least if the latter is an isolated zero of $f(x)-x$.

This conclusion immediately applies to the iteration problem, as any solution of it is a solution of Lüntz's problem, so that at least 
some information on this subject is obtained by Lüntz's way of attacking it.

II. Is the above assumption-namely that either $a_{1} \neq 0,1$ or $a_{1}=1, a_{2} \neq 0$-essential for the general behaviour of the results and especially for the equivalence of those problems?

This would seem to be presumable a priori: for it is evident that if the function $f(x)$ would reduce to $x$ itself, Lüntz's problem would become fully indeterminate, as the function $f(x)=x$ is reversible to every other one.

A most remarkable fact is that this presumption is not verified. Let the first term in (12) be reduced to $x$, the first following term which does not vanish being of degree $p$, so that

$$
f(x)=x+a_{p} x^{p}+\cdots \quad\left(p \geqq 2, a_{p} \neq 0\right) .
$$

Equating the coefficients of $x^{p}$ in both members of (11), we immediately see that $b_{1}=b_{1}^{p}$. Let us take, therefore,$^{7} b_{1}=1$, so that the expansion of $g(x)$ is also of the form

$$
g(x)=x+b_{1} x^{r}=\cdots \quad\left(r \geqq 2, b_{r} \neq 0\right) .
$$

If so, all terms issuing from the first term of (12) or from the first term of (13) annul each other. After them, the terms of lowest degree are in $x^{p+r-1}$, with coefficients respectively equal to $p a_{p} b_{r}$ and $r a_{p} b_{r}$ in both members, so that $r$ can not be different from $p$. Then, $b$ 's with suffixes greater than $p$ would be determined by equations some of which (except for $p=2$ ) will reduce to the form $a_{p} b_{p+q}=b_{p} a_{p+q}$ while, for $p=2$, we shall have

$$
a_{2} b_{3}-a_{3} b_{2}=a_{2} b_{2}\left(b_{2}-a_{2}\right) \text {. }
$$

We see that, in every case, the functions reversible to $f(x)$ and regular at $x=0$ constitute a one-parameter family, which necessarily coincides with the iterates of $f$.

We also see that Lüntz was right in supposing that two functions reversible to the same third one are reversible to each other. This, as is classic in the group theory, carries the consequence that the reversibility conditions can be expressed by the existence of invariants. Let $c_{1}$ be chosen once for all, different from 0 and 1: we can consider as a "reduced" form of $g(x)$ the one which has $c_{1}$ as coefficient of $x$. Every other coefficient in that reduced expansion will be deter-

7 Provisionally, we still exclude, like Lüntz, the value zero and, to simplify discussion, do not consider roots of unity. At any rate, in iteration problems it is natural (cf. Birkhoff, loc. cit. p. 9) to eliminate negative first coefficients. 
mined by these conditions and, on the other hand, we just saw that the reduced $g$ will be reversible not only to $f$, but also to any other function (13) reversible to $f$, so that such successive coefficients of the reduced expansion, when calculated in terms of the $a$ 's, must have the same values as the ones similarly calculated in terms of the $b$ 's: they give invariants (which, obviously, only seemingly depend on the special value of $c_{1}$ ). The first of them are

$$
\begin{aligned}
& j_{2}=a_{2}:\left(a_{1}-a_{1}^{2}\right), \quad j_{3}=\left(a_{3}-2 a_{2} j_{2}\right):\left(a_{1}-a_{1}^{3}\right), \\
& j_{4}=\left\{a_{4}-\left[\left(3 a_{1}+5\right) j_{3}+5 j_{2}^{2}\right] a_{2}\right\}:\left(a_{1}-a_{1}^{4}\right),
\end{aligned}
$$

Such invariants are evidently related to the coefficients of the infinitesimal transformation of the group-a connection which, however, we shall not investigate.

In the case, considered in II, of $a_{1}=1$, the first invariant, for $p=2$, is

$$
a_{3} / a_{2}-a_{2}
$$

while, for $p \geqq 2$, one or several invariants will be of the form $a_{p+q}: a_{p}$, after which there will come one analogous to (15).

III. The transformations $S: x \rightarrow f(x), T: x \rightarrow g(x)$, where $f$ and $g$ are connected by Lüntz's relation (11), being permutable to each other, the transform of $T$ by $S$ coincides with $T$. In other words, the curve $y=g(x)$ is invariant by the point transformation $X=f(x)$, $Y=f(y)$. The general problem of invariant points has been studied several times, especially by Poincaré, Sa muel Lattés, ${ }^{8}$ G. D. Birkhoff (loc. cit.) and the present author; but the present transformation behaves in an abnormal manner from this point of view, as there exist an infinite number of invariant curves, namely every $n$th iterate $y=f_{n}(x)$, such iterates existing at least for every integral $n$.

IV. The problem of reversible functions being connected with the iteration problem, we need not wonder at seeing it connected with Abel's equation (A). As a matter of fact, this connection is a very simple one. Let $\phi$ be any solution of (A) and let us apply the operation $\phi$ to both members of (11). We immediately see that the function

$$
\Phi(x)=\phi[g(x)]
$$

is again a solution of (A). Conversely, if $\phi$ and $\Phi$ be two solutions of

${ }^{8}$ Poincare, J. Math. Pures Appl. (4) vol. 1 (1885); Hadamard, Bull. Soc. Math. France vol. 29 (1901); Samuel Lattés, Annali di Matematica (3) vol. 13 (1907). 
(A), the function $g$ defined by (16)-in other words, $g(x)=\phi^{-1}[\Phi(x)]$ -is reversible to $f$. Any determinate function $g(x)$ permutable with $f(x)$ can be obtained in an infinite number of ways by this procedure.

V. We have noticed-see footnote (4)-that if we knew a finite Lie group $G$ containing the transformation $x \rightarrow f(x)$, we could deduce therefrom the one-parameter group (9) containing that same transformation. Now, in G. D. Birkhoff's example, the one-parameter group analogous to (9) does not exist (otherwise than formally). This would suggest that the corresponding transformation is contained in no finite Lie group. However, there is the objection that the deduction of (9) from $G$, by Elie Cartan's method, might be valid only in a local domain, Birkhoff's transformation lying beyond that domain.

VI. The case $a_{1}=0$, which Lüntz has explicitly excluded, is also implicitly excluded from Jabotinsky's study. Indeed, the impossibility of any regular solution of the form (9) at once appears from the first equation (10); as for any solution of that equation, the conditions $A_{1}(0)=1$ and $A_{1}(1)=0$ are contradictory. Therefore, no function which is regular around $x=0$ and whose expansion begins by a term of degree 2 or higher can belong to a regular continuous oneparameter group of Lie; nor can it, as we saw, belong to a regular finite group in any number of parameters.

Now, such a function $f$ of that kind being given, let us consider the reversibility problem. In the expansion (13) of a function $g$ reversible to $f$, we shall have, as previously, either

(1) $b_{1}=1$ : one easily sees that this gives nothing else than $g(x)$ identically equal to $x$; or

(2) $b_{1}=0$ : corresponding solutions are evidently $g(x)$ equal to $f(x)$ itself or to one of its iterates.

But there may be solutions other than these trivial ones, for the two functions $f(x)=x^{m}$ and $g(x)=x^{n}$ are obviously reversible. From those, or more generally from $f(x)=a x^{m}$ and $g(x)=b x^{n}$ (which are reversible if $a b^{m}=b a^{n}$ ), we obviously could deduce other ones seemingly, but not essentially, distinct from them by applying to $x, f(x)$ and $g(x)$ a common point transformation, regular and one-one around $x=0$. Whether this would afford the most general solution relating to our present case, $a_{1}=0$, remains to be investigated.

Another curious question would arise if equation (8) were to admit two different roots $x_{0}, x_{1}$ around which expansions were to behave differently from the expansions considered above-for example $f^{\prime}\left(x_{0}\right)=0, f^{\prime}\left(x_{1}\right)=0$-giving rise possibly to special kinds of singularities.

\section{COLUMBIA UNIVERSITY}

